\title{
Failure of random matrix theory to correctly describe quantum dynamics
}

\author{
Tsampikos Kottos ${ }^{1}$ and Doron Cohen ${ }^{2}$ \\ ${ }^{1}$ Max-Planck-Institut für Strömungsforschung, 37073 Göttingen, Germany \\ 2 Department of Physics, Harvard University, Cambridge, MA 02138
}

(April 2001)

\begin{abstract}
Consider a classically chaotic system which is described by a Hamiltonian $\mathcal{H}_{0}$. At $t=0$ the Hamiltonian undergoes a sudden-change $\mathcal{H}_{0} \mapsto \mathcal{H}$. We consider the quantummechanical spreading of the evolving energy distribution, and argue that it cannot be analyzed using a random-matrix theory (RMT) approach. RMT can be trusted only to the extend that it gives trivial results that are implied by first-order perturbation theory. Non-perturbative effects are sensitive to the underlying classical dynamics, and therefore the $\hbar \rightarrow 0$ behavior for effective RMT models is strikingly different from the correct semiclassical limit.
\end{abstract}

Consider a system whose total Hamiltonian is $\mathcal{H}=$ $\mathcal{H}(Q, P ; x)$, where $(Q, P)$ is a set of canonical coordinates, and $x$ is a constant parameter. We assume that the preparation and the representation of the system are determined by the Hamiltonian $\mathcal{H}_{0}=\mathcal{H}\left(Q, P ; x_{0}\right)$, and that both $\mathcal{H}_{0}$ and $\mathcal{H}$ generate classically chaotic dynamics of similar nature. Moreover, we assume that $\delta x \equiv\left(x-x_{0}\right)$ is classically small, meaning that it is possible to apply linear analysis in order to describe how the energy surfaces $\mathcal{H}(Q, P ; x)=E$ are deformed as a result of changing the value of $x$. Physically, going from $\mathcal{H}_{0}$ to $\mathcal{H}$ may signify a change of an external field, or switching on a perturbation, or sudden-change of effective-interaction (as in molecular dynamics). Quantum mechanically, we can use a basis where $\mathcal{H}_{0}=\mathbf{E}_{0}$ has a diagonal representation, while

$$
\mathcal{H}=\mathbf{E}_{0}+\delta x \mathbf{B}
$$

For reasonably small $\hbar$, it follows from general semiclassical considerations [1], that $\mathbf{B}$ is a banded matrix. Generically, this matrix looks random, as if its off-diagonal elements were independent random numbers.

It was the idea of Wigner [2] forty years ago, to study a simplified model, where the Hamiltonian is given by Eq. (11), and where $\mathbf{B}$ is a Banded Random Matrix (BRM) [3]. This approach is attractive both analytically and numerically. Analytical calculations are greatly simplified by the assumption that the off-diagonal terms can be treated as independent random numbers. Also from numerical point of view it is quite a tough task to calculate the true matrix elements of the $\mathbf{B}$ matrix. It requires a preliminary step where the chaotic $\mathcal{H}_{0}$ is diagonalized. Due to memory limitations one ends up with quite small matrices. We can think of Eq. (11) as describing fictitious motion on a lattice. For the model below (Eq. 2) we were able to handle $N=5000$ sites maximum. This should be contrasted with BRM simulations, where us- ing self-expanding algorithm [4] we were able to handle $N=100000$ sites along with significantly reduced CPU time.

However, the applicability of the RMT approach is a matter of conjecture. Obviously this conjecture should be tested. To be more specific, one should be aware that there is a hierarchy of challenges where the applicability of the RMT conjecture should be tested. Namely: The study of spectral statistics; The study of eigenstates; The study of quantum dynamics. While the issue of spectral statistics has become a major subject in "quantum chaos" studies [5], the two other issues are barely treated. In a previous study [7] we have demonstrated that the RMT approach is capable of giving the right qualitative picture of the parametric evolution of the eigenstates. As $\delta x$ is increased the eigenstates of Eq. (11) change in a qualitative agreement with Wigner's theory. Still, RMT fails to capture non-universal system-specific features.

In this Letter we turn to the study of quantum $d y$ namics. Here we are going to end up with a much more alarming claim. Namely, the RMT approach fails to give the correct dynamical picture. RMT can be trusted only to the extend that it gives trivial results that are implied by first-order perturbation theory. Non-perturbative effects are sensitive to the underlying classical dynamics, and therefore the $\hbar \rightarrow 0$ behavior for effective RMT models is strikingly different from the correct semiclassical limit. In this Letter we are going to establish the failure of RMT for the case of dynamics which (for $t>0$ ) is generated by a time independent Hamiltonian. This we hope paves the way towards making an analogous statement regarding the response of driven systems [6].

In order to test the RMT conjecture, we are going to use the same 'direct' approach and the same model as in Ref. [7]. For the first time we are going to compare the dynamics which is generated by a 'physical' Hamiltonian, with the corresponding dynamics that is obtained from an effective BRM model (EBRM). The latter is constructed by taking the matrix $\mathbf{B}$ of the 'physical' Hamiltonian, and then randomizing the signs of its off-diagonal elements. Such operation destroys any correlations between the matrix elements of $\mathbf{B}$, while keeping the band profile unaffected [8]. We study the Hamiltonian [7]

$$
\mathcal{H}(Q, P ; x)=\frac{1}{2}\left(P_{1}^{2}+P_{2}^{2}+Q_{1}^{2}+Q_{2}^{2}\right)+x \cdot Q_{1}^{2} Q_{2}^{2}
$$

with $x=x_{0}+\delta x$ and $x_{0}=1$. This Hamiltonian describes the motion of a particle in a $2 \mathrm{D}$ well $(2 \mathrm{DW})$. The units are chosen such that the mass is equal to one, the frequency for small oscillations is one, and for $\delta x=0$ the coefficient of the anharmonic term is also one. The 


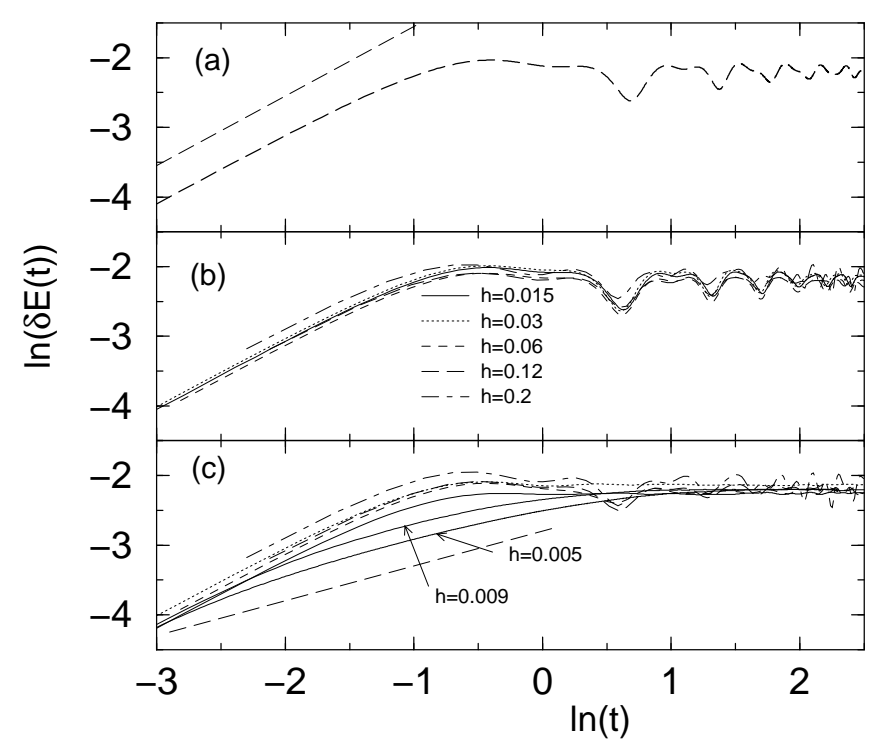

FIG. 1. (a) The classical energy spreading as a function of time. (b) The QM spreading for the 2DW Hamiltonian; (c) The QM spreading for the EBRM Hamiltonian. The energy in these simulations is $E \sim 3$, and $\delta x=0.2123$. In (a) we see a crossover from ballistic spreading $(\delta E \propto t)$ to saturation ( $\delta E \sim$ const $)$. Only one time scale $\left(\tau_{\mathrm{cl}} \sim 1\right)$ is involved. The light dashed line has slope 1 and is drawn to guide the eye. In (b) we see that the classical behavior is approached as $\hbar \rightarrow 0$. In (c) we see the opposite trend: as $\hbar \rightarrow 0$ an intermediate stage of diffusion $(\delta E \propto \sqrt{t})$ develops. (Here the light dashed line has slope $1 / 2)$. Different lines correspond to different values of $\hbar$ as in (b), and additional curves $(\hbar=0.009,0.005)$ have been added.

energy $E$ is the only dimensionless parameter of the classical motion. Our numerical study is focused on an energy window around $E \sim 3$ where the motion is mainly chaotic. Upon quantization we have a second dimensionless parameter, which is the scaled $\hbar$. Associated with $\hbar$ are two energy scales. One is the mean level spacing $\Delta \propto \hbar^{d}$ with $d=2$, and the other is the bandwidth $\Delta_{b} \propto \hbar$. The second scale is further discussed below.

It is useful to define a fluctuating quantity $\mathcal{F}(t) \equiv$ $-(\partial \mathcal{H} / \partial x)$ which for the 2DW model equals $\mathcal{F}(t)=$ $-Q_{1}^{2}(t) Q_{2}^{2}(t)$. The auto-correlation function of $\mathcal{F}(t)$ is denoted by $C(\tau)$. The associated correlation time is denoted by $\tau_{\mathrm{cl}}$. The power spectrum of the fluctuations $\tilde{C}(\omega)$ is the Fourier transform of $C(\tau)$. The band profile of the matrix $\mathbf{B}$ satisfies the semiclassical relation $\left|\mathbf{B}_{n m}\right|^{2} \approx(\Delta /(2 \pi \hbar)) \tilde{C}\left(\left(E_{n}-E_{m}\right) / \hbar\right)$. See Fig.2 of [7] for numerical demonstration. It is implied by this relation that the bandwidth is $\Delta_{b}=2 \pi \hbar / \tau_{\mathrm{cl}}$. For the quantum mechanical simulation the exact matrix $\mathbf{B}$ has been calculated numerically. Memory constraints limit the maximum size $(N)$ of the matrix that we can get. The EBRM Hamiltonian is obtained by randomizing the signs of the off-diagonal elements. A second, more 'loose' strategy, is to generate the EBRM B from scratch using the semiclassical band-profile as an input. The advantage of the latter strategy is that it opens the way to EBRM-model simulations with smaller $\hbar$, where the required $N$ is much

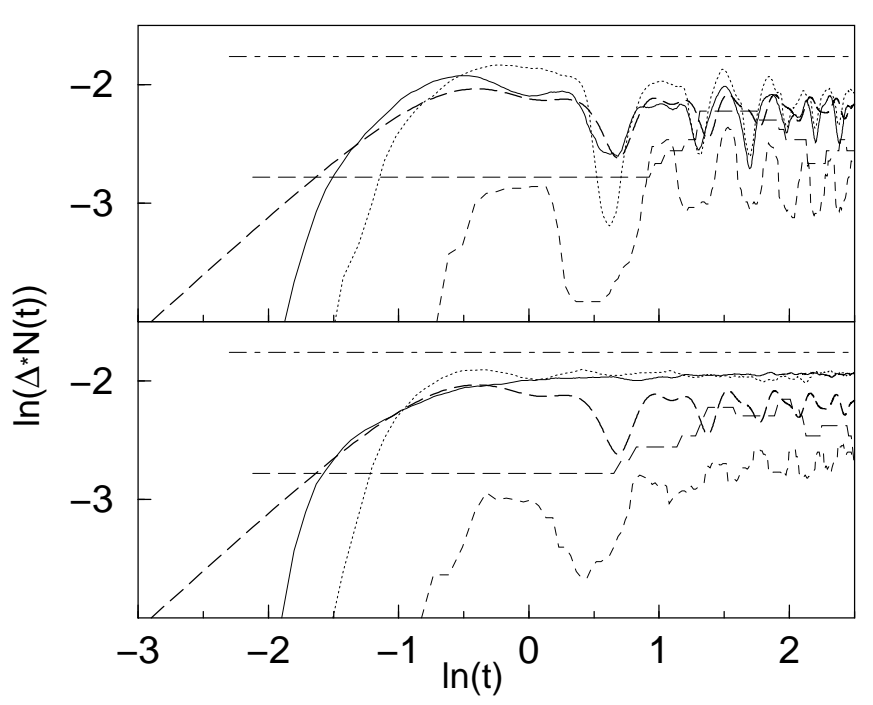

FIG. 2. The width $\Delta \times N(t)$ as a function of time. Different lines correspond to different $\hbar$-values as in Fig. 1b. The heavy dashed line is the classical $\delta E(t)$. Having separation of scales $(\Delta \times N(t) \ll \delta E(t))$ is an indication for having a perturbative spreading profile. The upper panel is for the $2 \mathrm{DW}$ Hamiltonian while the lower panel is for the EBRM model.

larger. We have verified that the latter strategy gives numerical results that agree with the sign-randomization approach.

The initial preparation is assumed to be microcanonical. This means, in the classical case, an ergodic distribution of initial 'points' on the energy surface $\mathcal{H}_{0}(Q, P)=$ $E$ with $E \sim 3$. In the quantum mechanical case we start each simulation with an initial eigenstate $m$ that has an energy $E_{m} \sim E$ where $2.75<E<3.2$. The time dependent evolution is determined by Schrodinger Equation. The probability distribution after time $t$ is $P_{t}(n \mid m)$. An average over initial state $(m)$ is taken in order to get the average profile $P_{t}(n-m)$. We characterize the evolving distribution using three different measures. The variance is $M(t)=\sum_{r} r^{2} P_{t}(r)$, or in energy units it is $\delta E(t)=\Delta \times \sqrt{M(t)}$. The width $N(t)$ is defined as the $r$ region that contains $50 \%$ of the probability. In case that we have a spreading profile that is characterized by a single energy scale, it is implied that $N(t)$ and $\sqrt{M(t)}$ would be the same (up to a numerical factor). The survival probability is $P(t)=P_{t}(r=0)$. The results of the simulations are presented in the Figs 1-4. The analysis of these results is discussed below.

Taking $\mathcal{H}$ to be a generator for the classical dynamics, the energy $E(t)=\mathcal{H}_{0}(Q(t), P(t))$ fluctuates. The fluctuations are characterized by the correlation time $\tau_{\mathrm{cl}}$, and by an amplitude $\delta E_{\mathrm{cl}}$. The initial preparation is assumed to be a microcanonical distribution that is supported by the energy-surface $\mathcal{H}_{0}(Q, P)=E(0)$. For $t>0$, the phase-space distribution spreads away from the initial surface. 'Points' of the evolving distribution move upon the energy-surfaces of $\mathcal{H}(Q, P)$. We are interested in the distribution of the energy $E(t)$ of the evolving 'points'. It is easily argued that for short times this distribution 


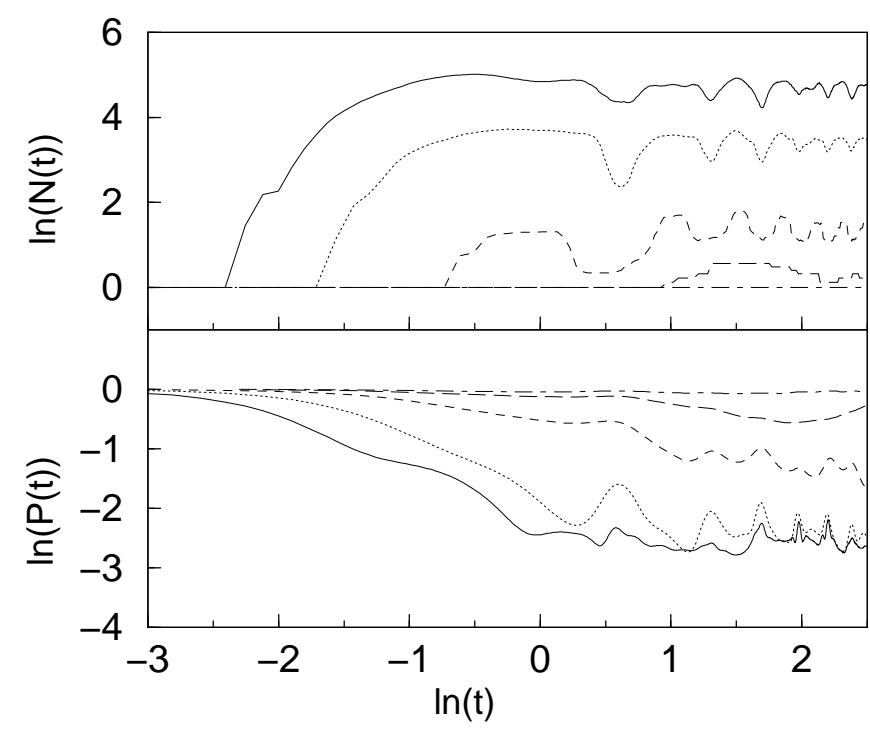

FIG. 3. The width $N(t)$ and the survival probability $P(t)$ for the 2DW model. Different lines correspond to different $\hbar$-values as in Fig. 1b. Having $N(t)=1$ or $P(t) \sim 1$ is the indication for having a standard perturbative spreading profile.

evolves in a ballistic fashion. Then, for $t \gg \tau_{\mathrm{cl}}$, due to ergodicity, a 'steady-state distribution' appears, where the evolving 'points' occupies an 'energy shell' in phasespace. The thickness of this energy shell equals $\delta E_{\mathrm{cl}}$. Thus we have a crossover from ballistic energy spreading to saturation. The dynamics in the classical limit is fully characterized by the two classical parameters $\tau_{\mathrm{cl}}$ and $\delta E_{\mathrm{cl}}$.

A quantitative description of the classical spreading is easily obtained. A straightforward derivation leads to the following result for the spreading

$$
\delta E_{\mathrm{cl}}(t)=\delta x \times \sqrt{2(C(0)-C(t))}
$$

As a particular result we get $\delta E_{\mathrm{cl}} \equiv \delta E_{\mathrm{cl}}(\infty)=$ $\delta x \sqrt{2 C(0)}$. The calculation of $\delta E_{\mathrm{cl}}(t)$ for the model Hamiltonian is presented in Fig. 1a. It is implied by Eq.(3) that the spreading $\delta E(t)$, from semiclassical point of view, is just a property of the band-profile. Thus, one may get to the wrong conclusion that models with the same band-profile should lead to the same $\delta E(t)$, provided the off-diagonal elements look random. If this were the case, it would be implied that the EBRM model would be equivalent to the $2 \mathrm{DW}$ model as far as the spreading $\delta E(t)$ is concerned. Looking on Fig.1 we see that this is not the case. As $\hbar \rightarrow 0$ the EBRM model further and further deviates from the (correct) semiclassical expectation.

In order to understand the observed results we would like to recall some of the theory of [9:7]. We already said that upon quantization we have the two energy scales $\Delta \propto \hbar^{d}$ and and $\Delta_{b} \propto \hbar$. Actually there is also a semiclassical energy scale $\Delta_{\mathrm{SC}} \propto \hbar^{2 / 3}$. Associated with these energy scales are three parametric scales $\delta x_{c}^{\mathrm{qm}} \ll \delta x_{\mathrm{prt}} \ll \delta x_{\mathrm{SC}}$, where the strong inequalities hold in the $\hbar \rightarrow 0$ limit. For the 2DW model, assuming $E \sim 3$ we have [7] the estimates $\delta x_{c}^{\mathrm{qm}} \approx 3.8 * \hbar^{3 / 2}$ and $\delta x_{\mathrm{prt}} \approx 5.3 * \hbar$ and $\delta x_{\mathrm{SC}} \approx 4 * \hbar^{2 / 3}$. In the standard perturbative regime $\left(\delta x<\delta x_{c}^{\mathrm{qm}}\right)$ the eigenstates of Eq.(1) have a simple perturbative structure. In the extended perturbative regime $\left(\delta x_{c}^{\mathrm{qm}}<\delta x<\delta x_{\mathrm{prt}}\right)$ the eigenstates of Eq.(11) have a core-tail structure that can be regarded as a generalization of Wigner's Lorentzian. In the non-perturbative regime $\left(\delta x>\delta x_{\mathrm{prt}}\right)$ the eigenstates have a purely non-perturbative structure. Depending on whether it is the EBRM Hamiltonian or the 2DW Hamiltonian, this 'ergodic' non-perturbative structure is either semicircle-like or semiclassical-like respectively. The semiclassical regime $\left(\delta x>\delta x_{\mathrm{SC}}\right)$ is contained in the non-perturbative regime. It is only there that we can trust detailed quantal-classical correspondence (QCC).

For the purpose of the present analysis it is convenient to specify the different regimes by regarding $\hbar$ as a free parameter. Thus, the standard perturbative regime is $\hbar>C_{\text {cqm }}$, the extended perturbative regime is $C_{\mathrm{prt}}<\hbar<C_{\mathrm{cqm}}$, and the non-perturbative regime is $\hbar<C_{\mathrm{prt}}$. The latter contains the semiclassical regime $\hbar<C_{\mathrm{SC}}$. Thus the semiclassical limit $\hbar \rightarrow$ 0 is a non-perturbative limit. In case of the $2 \mathrm{DW}$ model, the classical quantities are $C_{\text {cqm }}=0.41 * d x^{2 / 3}$ and $C_{\mathrm{prt}}=0.19 * d x$ and $C_{\mathrm{SC}}=0.12 * d x^{3 / 2}$. We have used in most of our numerical simulations $\delta x \sim 0.2$. Larger $\delta x$ may take us out of the classical linear regime. For this value of $\delta x$ we get $C_{\text {cqm }}=0.14$ and $C_{\text {prt }}=0.04$ and $C_{\mathrm{SC}}=0.01$. The smallest $\hbar$ value that we could allow without having memory-overflow was $\hbar=0.015$. This means that we were able to access the non-perturbative regime, though the semiclassical regime was out of reach.

As explained in [10] the essential features of the spreading behavior in the perturbative regimes can be analyzed using first order perturbation theory (FOPT). Since correlations between off-diagonal elements are not important for FOPT, it follows that the EBRM-model and the 2DW model should be trivially equivalent in such case. It is only in the non-perturbative regime where the question of their equivalence becomes non-trivial. In case of the standard BRM we have witnessed [10] in the non-perturbative regime a premature departure from ballistic behavior, and appearance of an intermediate diffusive stage. We observe essentially the same behavior in case of the EBRM Hamiltonian (Fig. 1c). But with the 2DW Hamiltonian (Fig. 1b) we do not have such an effect: As $\hbar \rightarrow 0$ the correspondence with the classical behavior becomes better and better. Thus our simulations demonstrate that having diffusion in the nonperturbative regime is an artifact of the RMT approach. In our previous work [10] we did not have a numerical proof to support such a strong statement. There, all we were able to do, was to argue that RMT should fail in the deep semiclassical regime ( $\hbar \ll C_{\mathrm{SC}}$ ), thus leaving open the possibility for having an intermediate regime $\left(C_{\mathrm{SC}}<\hbar<C_{\mathrm{prt}}\right)$ where RMT might be valid. As we 


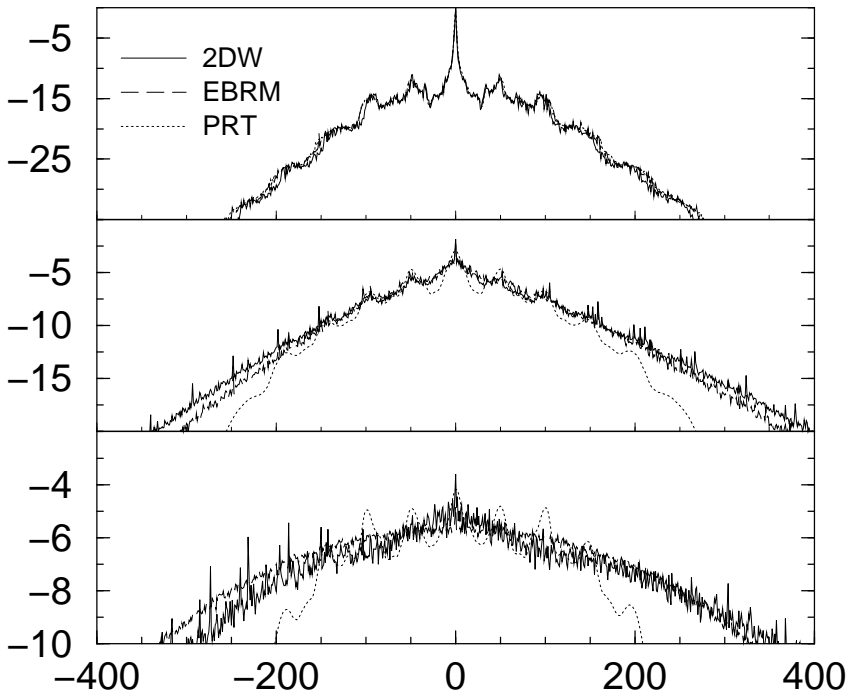

FIG. 4. The spreading profile $P_{\infty}(r)$ in representative cases. The upper panel is an example for a standard perturbative profile $(P(t) \sim 1)$. The middle panel is an example for a perturbative core-tail structure $(\Delta \times N \ll \delta E)$. The lower panel is an example for an ergodic-like non-perturbative structure $(\Delta \times N \sim \delta E)$.

see, our numerical results do not give any indication for the existence of such intermediate regime. The failure of RMT happens as soon as we enter the non-perturbative regime $\left(\hbar<C_{\mathrm{prt}}\right)$.

As we make $\hbar$ smaller there is no indication in Fig. 1 for entering a non-perturbative regime. In [9, 7, 10] we have made the important distinction between detailed $\mathrm{QCC}$ and restricted QCC. The former pertains to the whole spreading profile, while the latter pertains only to the variance. Restricted QCC is a robust type of correspondence that does not require a 'very small $\hbar$ '. If we want to have an indication for the crossover to a non-perturbative behavior we should look on other measures, such as $N(t)$ of Fig. 2. If the spreading were classical-like, it would imply that the spreading profile is characterized by a single energy scale. In such case we would expect that $N(t)$ and $\sqrt{M(t)}$ would be the same (up to a numerical factor). Indeed this is the case for the $\hbar<0.04$ runs of Fig.2. However, this is definitely not the case in the perturbative regimes, where we have a separation of energy scales $N(t) \ll \sqrt{M(t)}$. In the perturbative regimes $M(t)$ is determined by the tails, and it is insensitive to the size of the 'core' region. The width $N(t)$ constitutes a practical estimate for the latter (see Fig.3a). It is $N(t)=1$ for a standard perturbative profile, and $1 \ll N(t) \ll \sqrt{M(t)}$ for a fully developed core-tail structure. An alternate way to identify a standard perturbative profile is via the survival probability $P(t)$. Indeed for $\hbar<0.14$ we see in Fig.3b that we have $P(t) \sim 1$.

The difference between the perturbative and the nonperturbative spreading profiles is further illustrated in Fig.4. Here we have plotted representative average saturation profiles, along with a comparison with the per- turbative core-tail calculation (PRT for brevity). The saturation profile is given by the expression $P_{\infty}(n \mid m)=$ $\sum_{n^{\prime}}\left|\left\langle n\left(x_{0}\right) \mid n^{\prime}(x)\right\rangle\right|^{2}\left|\left\langle n^{\prime}(x) \mid m\left(x_{0}\right)\right\rangle\right|^{2}$. It can be regarded as the auto-convolution of $P_{\mathrm{E}}(n \mid m)=\left|\left\langle n(x) \mid m\left(x_{0}\right)\right\rangle\right|^{2}$. Thus the average saturation profile $P_{\infty}(r)$ is approximately related to the average local density of states $P_{\mathrm{E}}(r)$. The latter has been analyzed in [7]. In Fig.3 we have calculated the PRT of $P_{\infty}(r)$ via an autoconvolution of the PRT of $P_{\mathrm{E}}(r)$. In the extended perturbative regime the major features of the saturation profile are captures by the PRT. The differences are mainly in the far tails where higher order perturbation theory is essential. There are also differences in the small scale details, where the non-perturbative mixing is important. In the non-perturbative regime the saturation profile becomes purely non-perturbative, and the PRT becomes useless. This is because there is no longer separation of energy scales, which is the working assumption of the core-tail theory.

We thank Felix Izrailev for suggesting to study the 2DW model.

[1] M. Feingold and A. Peres, Phys. Rev. A 34 591, (1986). M. Feingold, D. Leitner, M. Wilkinson, Phys. Rev. Lett. 66, 986 (1991).

[2] E. Wigner, Ann. Math 62548 (1955); 65203 (1957); Y.V. Fyodorov, O.A. Chubykalo, F.M. Izrailev and G. Casati, Phys. Rev. Lett. 76, 1603 (1996).

[3] G. Casati, B.V. Chirikov, I. Guarneri, F.M. Izrailev, Phys. Rev. E 48, R1613 (1993); Phys. Lett. A 223, 430 (1996).

[4] F. M. Izrailev, T. Kottos, A. Politi and G. P. Tsironis, Phys. Rev. E., 55, 4951 (1997).

[5] In most of the RMT literature (including the later works by Wigner himself), it is assumed that for the purpose of 'quantum chaos' studies one can consider full (rather than banded) matrices, and the first term $\mathbf{E}_{0}$ is generally neglected. In spite of these enormous simplifications, it turns out that the so-called Gaussian invariant ensembles (GOE,GUE) provide a valid description of some major spectral properties.

[6] D. Cohen and T. Kottos, Phys. Rev. Lett. 85, 4839 (2000).

[7] D. Cohen and T. Kottos, Phys. Rev. E, 63 36203, (2001).

[8] Note that the standard BRM model, unlike our EBRM model, involves an additional simplification. Namely, in case of the former model one further assumes that $\mathbf{B}$ has a rectangular band profile.

[9] D. Cohen, Phys. Rev. Lett. 82, 4951 (1999); Ann. Phys. 283, 175-231 (2000). D. Cohen and E.J. Heller, Phys. Rev. Lett. 84, 2841 (2000). D. Cohen, A. Barnett and E.J. Heller, Phys. Rev. E 63, 46207 (2001). D. Cohen, in Proceedings of the International School of Physics 'Enrico Fermi' Course CXLIII "New Directions in Quantum Chaos", Edited by G. Casati, I. Guarneri and U. Smilansky, IOS Press, Amsterdam, 2000.

[10] D. Cohen, F.M. Izrailev and T. Kottos, Phys. Rev. Lett. 842052 (2000). 